PROCEEDINGS OF THE

AMERICAN MATHEMATICAL SOCIETY

Volume 125, Number 6, June 1997, Pages 1737-1744

S 0002-9939(97)03804-5

\title{
NON-EXISTENCE AND UNIQUENESS RESULTS FOR BOUNDARY VALUE PROBLEMS FOR YANG-MILLS CONNECTIONS
}

\author{
TAKESHI ISOBE
}

(Communicated by Ronald Stern)

\begin{abstract}
We show uniqueness results for the Dirichlet problem for YangMills connections defined in $n$-dimensional $(n \geq 4)$ star-shaped domains with flat boundary values. This result also shows the non-existence result for the Dirichlet problem in dimension 4, since in 4-dimension, there exist countably many connected components of connections with prescribed Dirichlet boundary value. We also show non-existence results for the Neumann problem. Examples of non-minimal Yang-Mills connections for the Dirichlet and the Neumann problems are also given.
\end{abstract}

\section{INTRODUCTION}

Let $M$ be a Riemannian manifold with boundary and $G$ a compact Lie group. Let $A_{0}$ be a given smooth connection defined on a principal $G$-bundle $P_{0} \rightarrow \partial M$. We denote by $\mathcal{A}\left(A_{0}\right)$ the space of smooth connections defined in principal $G$-bundles over $M$ with Dirichlet boundary value $A_{0}$ at $\partial M$. That is,

$$
\begin{aligned}
\mathcal{A}\left(A_{0}\right)=\{ & \{A: A \text { is a smooth connection defined in some } \\
& \text { principal } \left.G \text {-bundle over } M \text { with } i^{*} A \sim A_{0} \text { over } \partial M\right\},
\end{aligned}
$$

where $i^{*} A \sim A_{0}$ means that $i^{*} A$ is gauge equivalent to $A_{0}$ over $\partial M$ and $i: \partial M \hookrightarrow M$ is the inclusion map.

By definition, a connection $A$ is a solution to the Dirichlet problem for YangMills equations defined in $M$ with boundary value $A_{0}$ at $\partial M$ if $A \in \mathcal{A}\left(A_{0}\right)$ with finite energy and $A$ is Yang-Mills, that is, $D_{A}^{*} F_{A}=0$ in $M$. Here $D_{A}^{*}$ is the formal adjoint of the covariant exterior derivative $D_{A}=d+[A$,$] with respect to the L^{2}$ metric on $\Lambda^{2} T^{*} M \otimes A d(P)$ induced from the Riemannian metric on $M$ and adjoint invariant metric on $\mathfrak{g}$, the Lie algebra of $G$.

We also recall the definition of the Neumann problem. A connection $A$ is a solution to the Neumann problem for Yang-Mills equations if and only if $A$ is YangMills in $M$ with finite energy and $i^{*}\left(* F_{A}\right)=0$ on $\partial M$. Here $*: \Lambda^{2} T^{*} M \otimes \operatorname{Ad}(P) \rightarrow$ $\Lambda^{2} T^{*} M \otimes \operatorname{Ad}(P)$ is the Hodge star operator.

Dirichlet and Neumann problems for Yang-Mills connections were first defined and studied by Marini [7]. In [7], Marini showed the existence and regularity of

Received by the editors December 8, 1995.

1991 Mathematics Subject Classification. Primary 35J50, 58E15, 81T13.

Key words and phrases. Yang-Mills, boundary value problems, non-existence, uniqueness.

(C)1997 American Mathematical Society 
absolute minimum solutions for the Dirichlet problem, and Neumann problem with prescribed class $\eta \in H^{2}\left(M ; \pi_{1}(G)\right)$, when $\operatorname{dim} M=4$.

In [4], Isobe and Marini studied the existence of "topologically distinct solutions" to the Dirichlet problem when $M=B^{4}=\left\{x \in \mathbb{R}^{4}:|x| \leq 1\right\}$ and $G=S U(2)$ using the fact that in this case $\mathcal{A}\left(A_{0}\right)$ is the disjoint union of infinitely many connected components $\mathcal{A}_{k}$ indexed by $k \in \mathbb{Z}$ (specifically, for some fixed $B \in \mathcal{A}\left(A_{0}\right), \mathcal{A}_{k}=$ $\{A: C(B)-C(A)=k\}$, where $\left.C(A)=\frac{1}{8 \pi^{2}} \int_{M} \operatorname{tr}\left(F_{A} \wedge F_{A}\right) * 1\right)$. It is shown that for "generic boundary values", there exist infinitely many topologically distinct solutions, and for any non-flat boundary values, there exist at least two topologically distinct solutions. These solutions all minimize the action on their component. In [4] we also showed that for some boundary values $A_{0}$ the action attains its infimum on only finitely many components, and hence does not attain its infimum on infinitely many others.

But the following question remained for the Dirichlet problem: Is there a YangMills connection (not necessarily minimizing) in each component of $\mathcal{A}\left(A_{0}\right)$ ?

In this paper, we show that for a flat boundary value $A_{0}$, a flat connection is the only solution for the Dirichlet problem with boundary value $A_{0}$ when the base manifold is star-shaped (see $\S 2$ for the definition) and structure group $G$ is an arbitrary compact Lie group. Thus we cannot, in general, expect the existence of Yang-Mills connections in each connected component of $\mathcal{A}\left(A_{0}\right)$.

We also show that this uniqueness result does not hold for general $M$. We give an example of $M$ (annulus) such that there exists a non-flat Yang-Mills connection on some principal bundle over $M$ which is flat at $\partial M$. This connection is necessarily a non-minimal Yang-Mills connection. The construction of this connection comes from the one given in [9], where Parker constructed non-minimal Yang-Mills connections over $S^{4}$ or $S^{3} \times S^{1}$. However, in our case, the argument is simplified by using a direct variational method.

As for the Neumann problem, if $\eta \in H^{2}\left(M ; \pi_{1}(G)\right)$ is trivial, the solution obtained by Marini [7] is a flat connection. This raises the following problem: Does there exist a non-flat connection for the Neumann problem if $\eta \in H^{2}\left(M ; \pi_{1}(G)\right)$ vanishes?

We show in this paper, when $\operatorname{dim} M=4$, for star-shaped domains, a flat connection is the only solution for the Neumann problem. We also give an example of a non-flat Yang-Mills connection on some principal $S U(2)$-bundle $P$ with $\eta(P)=0$ which satisfies the Neumann condition.

See also [14] for a uniqueness result to the Dirichlet problem for (anti-)self-dual connections. Note that we do not restrict ourselves to (anti-)self dual connections.

Our result also shows similarities between our results and other non-existence (or uniqueness) results related to Yamabe equations ([10]), harmonic mappings ([6]), constant mean curvature equations ([17]), etc...

\section{Proof of the MAin Results}

Our main results are the following. The first result is concerned with the Dirichlet problem:

Theorem 2.1. (1) Let $n \geq 5, M$ a $C^{2}$-star-shaped bounded domain in $\mathbb{R}^{n}$ with flat metric, and $G$ a compact Lie group. Let $A_{0}$ be a flat connection on some principal $G$-bundle $P_{0} \rightarrow \partial M$. Assume $A \in \mathcal{A}\left(A_{0}\right)$ is a solution to the Dirichlet problem 
for Yang-Mills connections. Then $A$ is a flat connection, that is, the curvature $F_{A}=d A+A \wedge A$ of $A$ vanishes.

(2) Let $n=4$ and $G$ be as in (1). Assume $M$ is a $C^{2}$-strictly star-shaped bounded domain in $\mathbb{R}^{4}$ with flat metric. Then the same conclusion as in (1) holds.

Our next result is concerned with the Neumann problem:

Theorem 2.2. Let $n=4$ and $G$ a compact Lie group. Let $M$ be a $C^{2}$-strictly star-shaped bounded domain in $\mathbb{R}^{4}$ with flat metric. Assume $A$ is a solution to the Neumann problem for Yang-Mills connections. Then $A$ is a flat connection.

Before we give the proofs of the above theorems, we give here the definitions of star-shaped and strictly star-shaped domains.

Definition 2.3. (1) A domain $M \subset \mathbb{R}^{n}$ is called star-shaped if there exists a point $x_{0} \in M$ such that the line segment $\overline{x x_{0}}$ is contained in $M$ for all $x \in M$. In this case, we have $\left\langle x-x_{0}, \nu(x)\right\rangle \geq 0$ for any point $x \in \partial M$, where $\nu(x)$ is the outer normal at $x \in \partial M$ and $\langle\cdot, \cdot\rangle$ is the inner product in $\mathbb{R}^{n}$.

(2) A domain $M \subset \mathbb{R}^{n}$ is called strictly star-shaped if $M$ is star-shaped and $\left\langle x-x_{0}, \nu(x)\right\rangle>0$ for any $x \in \partial M$, where $x_{0}$ is as in (1).

Note that the star-shaped domain is contractible. Therefore for such base manifold $M$ and any compact Lie group $G, \eta \in H^{2}\left(M ; \pi_{1}(G)\right)$ is always trivial.

Proof of the Theorem 2.1 and Theorem 2.2. Both theorems follow from the following first variation formula for Yang-Mills fields (see [11]):

Lemma 2.4. Let $\left\{e_{1}, \ldots, e_{n}\right\}$ be an orthonormal tangent frame for $T M$. Let $A$ be a solution to the Dirichlet or the Neumann problem for Yang-Mills equations. Then the following holds for any vector field $X$ in $M$ with compact support in $M^{\circ}$ :

$$
\int_{M}\left|F_{A}\right|^{2} \operatorname{div} X-4\left(F_{A}\left(\nabla_{e_{i}} X, e_{j}\right), F_{A}\left(e_{i}, e_{j}\right)\right)=0 .
$$

Here $(\cdot, \cdot)$ in the above equation is the adjoint invariant inner product of $\mathfrak{g}$.

In [11] this result is stated for Yang-Mills connections without boundary conditions, however, this is also true for Yang-Mills connections with boundary value conditions, since the variation used in [11] does not change boundary values.

We first prove Theorem 2.1.

Without loss of generality we may assume that $0 \in M$ and $M$ is (strictly) starshaped with respect to the point $x_{0}=0$ (see Definition 2.3).

For $\delta>0$, define

$$
\mathcal{O}_{\delta}(\partial M):=\{x \in M: d(x, \partial M)<\delta\} .
$$

Since $\partial M$ is $C^{2}$, there exists $\delta>0$ such that the map $\pi: \mathcal{O}_{\delta}(\partial M) \rightarrow \mathbb{R}$ defined by $\pi(x)=d(x, \partial M)$ is $C^{1}$.

For such $\delta$ define the map $\Pi: M \rightarrow \mathbb{R}$ as

$$
\Pi(x):= \begin{cases}\pi(x) & \text { if } x \in \mathcal{O}_{\delta}(\partial M), \\ \delta & \text { if } x \in M \backslash \mathcal{O}_{\delta}(\partial M) .\end{cases}
$$

$\Pi$ is a Lipschitz function defined in $M$. 
Next define the map $\rho_{\epsilon}: \mathbb{R} \rightarrow \mathbb{R}$ for $\epsilon>0$ as

$$
\rho_{\epsilon}:= \begin{cases}0 & \text { if } x \leq \epsilon, \\ 1 & \text { if } x \geq 2 \epsilon, \\ \frac{x}{\epsilon}-1 & \text { if } \epsilon \leq x \leq 2 \epsilon .\end{cases}
$$

For $\epsilon>0$ with $2 \epsilon<\delta$, define the vector field $X_{\epsilon}$ in $M$ by

$$
X_{\epsilon}=\rho_{\epsilon}(\Pi(x)) \sum_{i=1}^{n} x_{i} \frac{\partial}{\partial x_{i}} .
$$

Note that the vector field $X_{\epsilon}$ is only Lipschitzian, and the formula (2.1) holds for Lipschitzian vector fields by a density argument. Also note that $\operatorname{supp}\left(X_{\epsilon}\right) \subset\{x \in$ $M: \Pi(x) \geq \epsilon\} \subset M^{\circ}$.

We insert this vector field $X_{\epsilon}$ in the first variational formula (2.1), taking $e_{i}=$ $\partial / \partial x_{i}$. A short calculation gives

$$
\begin{aligned}
0= & (n-4) \int_{M} \rho_{\epsilon}(\Pi(x))\left|F_{A}\right|^{2} d x+\int_{M} \dot{\rho}_{\epsilon}(\Pi(x))\langle\nabla \Pi(x), x\rangle\left|F_{A}\right|^{2} d x \\
& -4 \int_{M} \dot{\rho}_{\epsilon}(\Pi(x))\left(F_{A}\left(x_{k} \frac{\partial}{\partial x_{k}}, \frac{\partial}{\partial x_{j}}\right), F_{A}\left(\nabla \Pi(x), \frac{\partial}{\partial x_{j}}\right)\right) d x .
\end{aligned}
$$

Here and in the following, we use the summation convention.

Letting $\epsilon \downarrow 0$ in (2.3), we obtain

$$
\begin{aligned}
0= & (n-4) \int_{M}\left|F_{A}\right|^{2} d x-\int_{\partial M}\langle x, \nu(x)\rangle\left|F_{A}\right|^{2} \\
& +4 \int_{\partial M}\left(F_{A}\left(x_{k} \frac{\partial}{\partial x_{k}}, \frac{\partial}{\partial x_{j}}\right), F_{A}\left(\frac{\partial}{\partial \nu}, \frac{\partial}{\partial x_{j}}\right)\right) .
\end{aligned}
$$

We rewrite (2.4) using the new tangent frame $\left\{\nu, \tau_{1}, \ldots, \tau_{n-1}\right\}$ at $\partial M$. Here $\left\{\tau_{1}, \ldots, \tau_{n-1}\right\}$ is an orthonormal tangent frame of $\partial M$. Then $\sum_{k=1}^{n} x_{k} \frac{\partial}{\partial x_{k}}=$ $\langle x, \nu\rangle \frac{\partial}{\partial \nu}+\sum_{k=1}^{n-1}\left\langle x, \tau_{k}\right\rangle \frac{\partial}{\partial \tau_{k}}$, and (2.4) becomes

$$
\begin{aligned}
0= & (n-4) \int_{M}\left|F_{A}\right|^{2} d x-\int_{\partial M}\langle x, \nu\rangle\left|F_{A}\right|^{2}+4 \int_{\partial M}\langle x, \nu\rangle\left|F_{A}\left(\nu, \tau_{k}\right)\right|^{2} \\
& +4 \int_{\partial M}\left\langle x, \tau_{k}\right\rangle\left(F_{A}\left(\tau_{k}, \tau_{l}\right), F_{A}\left(\nu, \tau_{l}\right)\right) .
\end{aligned}
$$

By the Dirichlet boundary condition $i^{*} A \sim 0$, we have $F_{A}\left(\frac{\partial}{\partial \tau_{k}}, \frac{\partial}{\partial \tau_{l}}\right)=0$ for all $1 \leq k, l \leq n-1$. Thus from (2.5), we get

$$
(n-4) \int_{M}\left|F_{A}\right|^{2} d x-\int_{\partial M}\langle x, \nu\rangle\left|F_{A}\right|^{2}+4 \int_{\partial M}\langle x, \nu\rangle\left|i^{*}\left(* F_{A}\right)\right|^{2}=0 .
$$

Using again the condition $i^{*} A \sim 0$, we reduce (2.6) to the following form:

$$
(n-4) \int_{M}\left|F_{A}\right|^{2} d x+3 \int_{\partial M}\langle x, \nu\rangle\left|i^{*}\left(* F_{A}\right)\right|^{2}=0 .
$$

When $n>4$ and $M$ is star-shaped, (2.7) implies $F_{A}=0$. This is the assertion of Theorem 2.1 (1).

To prove Theorem 2.1. (2), we work more.

Under the assumption of Theorem 2.1 (2) we have, by $(2.7), i^{*}\left(* F_{A}\right)=0$ on $\partial M$. Combining this with the Dirichlet boundary condition, we conclude that all components of $F_{A}$ vanish on $\partial M$. 
Let $\rho>0$ be such that the nearest point retraction $r: \mathcal{O}_{\rho}(M) \rightarrow \bar{M}$ defined by $r(x)=x$ if $x \in M$ and $r(x)=y_{x}$ if $x \in \mathcal{O}_{\rho}(M) \backslash M$, where $y_{x} \in \partial M$ is the unique point satisfying $d(x, M)=d\left(x, y_{x}\right)$, is well defined. Here $\mathcal{O}_{\rho}(M):=$ $\left\{x \in \mathbb{R}^{n}: d(x, M)<\rho\right\}$.

We define the connection $\tilde{A}$ defined in $\mathcal{O}_{\rho}(M)$ by $\tilde{A}=r^{*} A$. Note that $F_{\tilde{A}}=0$ on $\mathcal{O}_{\rho}(M) \backslash M$.

We need the following lemma.

Lemma 2.5. $\tilde{A}$ is a (weak) Yang-Mills connection in $\mathcal{O}_{\rho}(M)$.

Proof. First note that

$$
F_{\tilde{A}} \in L^{2}\left(\Lambda^{2} T^{*} \mathcal{O}_{\rho}(M) \otimes A d\left(r^{*} P\right)\right) \quad \text { and } \quad \tilde{A} \in L_{1}^{2}\left(T^{*} \mathcal{O}_{\rho}(M) \otimes A d\left(r^{*} P\right)\right) .
$$

We need to show the following:

$$
\begin{array}{cl}
\int_{\mathcal{O}_{\rho}(M)}\left(F_{\tilde{A}}, D_{\tilde{A}} \varphi\right)=0 & \text { for all } \varphi \in C^{\infty}\left(T^{*} \mathcal{O}_{\rho}(M) \otimes \operatorname{Ad}\left(r^{*} P\right)\right) \\
& \text { with } \operatorname{supp}(\varphi) \subset \mathcal{O}_{\rho}(M) .
\end{array}
$$

Fix such $\varphi \in C^{\infty}\left(T^{*} \mathcal{O}_{\rho}(M) \otimes A d\left(r^{*} P\right)\right)$. We have

$$
\begin{aligned}
\int_{\mathcal{O}_{\rho}(M)}\left(F_{\tilde{A}}, D_{\tilde{A}} \varphi\right) & =\int_{\mathcal{O}_{\rho}(M) \backslash \bar{M}}\left(F_{\tilde{A}}, D_{\tilde{A}} \varphi\right)+\int_{M}\left(F_{\tilde{A}}, D_{\tilde{A}} \varphi\right) \\
& =\int_{M}\left(F_{\tilde{A}}, D_{\tilde{A}} \varphi\right),
\end{aligned}
$$

since $F_{\tilde{A}}=0$ in $\mathcal{O}_{\rho}(M) \backslash \bar{M}$. On the other hand, since $D_{\tilde{A}}^{*} F_{\tilde{A}}=D_{\tilde{A}}^{*} F_{A}=0$ in $M$ and all components of $F_{\tilde{A}}$ vanish on $\partial M$, by integration by parts, we have

$$
\int_{M}\left(F_{\tilde{A}}, D_{\tilde{A}} \varphi\right)=0 .
$$

Combining (2.8) and (2.9), we complete the proof.

We continue the proof of Theorem 2.1 (2).

By the regularity theory for weak Yang-Mills connections over 4-manifolds [15], there exists a gauge $g \in L_{2}^{2}\left(\mathcal{O}_{\rho}(M) ; G\right)$ such that $\hat{A}:=g^{*} A \in C^{\infty}$. Since $\hat{A}$ is a Yang-Mills connection in $\mathcal{O}_{\rho}(M)$, and by the Bianchi identity, it satisfies $\left(D_{\hat{A}} D_{\hat{A}}^{*}+D_{\hat{A}}^{*} D_{\hat{A}}\right) F_{\hat{A}}=0$.

On the other hand, since $F_{\tilde{A}}=0$ in $\mathcal{O}_{\rho}(M) \backslash \bar{M}$, by the unique continuation theorem applied to the 2nd order elliptic partial differential operator $D_{\hat{A}} D_{\hat{A}}^{*}+$ $D_{\hat{A}}^{*} D_{\hat{A}}[1]$, we have $F_{\tilde{A}}=0$ in $\mathcal{O}_{\rho}(M)$. Thus $F_{A}=0$ in $M$ and $A$ is a flat connection. This completes the proof of Theorem 2.1 (2).

Next we prove Theorem 2.2.

By (2.5) and the Neumann condition $i^{*}\left(* F_{A}\right)=0$ on $\partial M$, we have

$$
(n-4) \int_{M}\left|F_{A}\right|^{2} d x-\int_{\partial M}\langle x, \nu\rangle\left|F_{A}\right|^{2}=0 .
$$

$n=4,\langle x, \nu\rangle>0$ on $\partial M$ and (2.10) imply $F_{A}=0$ on $\partial M$. The same argument in the case of Theorem 2.1 (2) implies $F_{A}=0$ in this case. This completes the proof. 


\section{EXAMPLES}

In this section, we give an example of a principal $S U(2)$-bundle $P \rightarrow M$ such that the Dirichlet problem

$$
\left\{\begin{array}{lll}
D_{A}^{*} F_{A} & =0 & \text { in } \quad M, \\
i^{*} A & \sim 0 & \text { on } \quad \partial M
\end{array}\right.
$$

has a non-flat solution. We also construct an example of a non-flat Yang-Mills connection for the Neumann problem when $\eta(P) \in H^{2}\left(M ; \pi_{1}(G)\right)$ vanishes. Our example is constructed for $M=$ cylindrical domain in dimension 4 or, by conformal invariance, for $M=$ annular domain $\subset \mathbb{R}^{4}$. The construction is based on the work of Parker [9]. See also [5], [16].

Let $M=S^{3} \times[0,1]$. Identify $S^{3}$ with $S U(2)$. With this identification, $S U(2)$ acts $S^{3}$ from right and left. This action extends, in the obvious way, to an action on the trivial $S U(2)$ bundle over $M$. It is not hard to show (see [9] for details) that any biinvariant connection on $M$ is gauge equivalent to one of the form

$$
A=\left(\frac{x(t)+1}{2}\right) \sum e_{i} \otimes e^{i},
$$

where $\left\{e^{i}\right\}$ and $\left\{e_{i}\right\}$ are dual left-invariant bases of $T^{*} S^{3}$ and $T S^{3}$ and $x(t)$ is a real-valued function on $[0,1]$. When this connection is restricted to the sphere $S^{3} \times\{t\}$, its curvature is

$$
F_{i j}=\left(\frac{x(t)^{2}-1}{4}\right)\left[e_{i}, e_{j}\right] .
$$

We can then express the Yang-Mills action in terms of $x(t)$ (again, see [9]):

$$
\mathcal{Y} \mathcal{M}(A)=\frac{3}{4} \int_{0}^{1}\left|\frac{d x}{d t}\right|^{2}+\left(x^{2}-1\right)^{2} d t
$$

By Palais' symmetric criticality principle, a critical point of this functional is a Yang-Mills field (see [8], [9]).

We first construct an example for the Dirichlet problem. Note that the connection (3.1) is flat on $\partial M$ if $x(0)=-1$ and $x(1)=1$. Thus we seek critical points of $\mathcal{Y} M$ under these boundary conditions. But these are easily found: direct methods in the calculus of variations show that the functional (3.3) has a minimizer on the Hilbert space

$$
H_{b}^{1}=\left\{x \in L^{2}([0,1]): x^{\prime} \in L^{2}([0,1]), x(0)=-1, x(1)=1\right\} .
$$

This minimizer is a smooth solution of $x^{\prime \prime}=x\left(x^{2}-1\right)$ with $x(0)=-1$ and $x(1)=1$.

We claim that the connection $A$ corresponding to this $x$ is a non-flat connection with flat boundary value. To prove this, we only need to show that $A$ is not a flat connection. Suppose $A$ is flat; then $\frac{d x}{d t}=0$ and $x^{2}=1$. But these contradict the boundary conditions $x(0)=-1$ and $x(1)=1$. Thus we complete the proof of our claim.

Next we construct an example for the Neumann problem. Notations are the same for the Dirichlet case. First, note that $\eta(P)=0$ since $H^{2}\left(M ; \pi_{1}(S U(2))\right)=0$. Since the curvature of $A$ is given by $F_{A}=d t \wedge \frac{d A}{d t}+F_{A}^{S^{3}}$, where $F_{A}^{S^{3}}$ is the curvature of the connection $A(t)$ on $S^{3} \times\{t\}$, the Neumann condition is equivalent to the condition $\frac{d x}{d t}(0)=\frac{d x}{d t}(1)=0$. Thus we need to find a solution of the equation $\frac{d^{2} x}{d t^{2}}=x\left(x^{2}-1\right)$ with $\frac{d x}{d t}(0)=\frac{d x}{d t}(1)=0$. This has a trivial solution $x \equiv 0$. The 
connection $A$ corresponding to this trivial solution is a solution for the Neumann problem and this is not flat since we have $\mathcal{Y M}(A)=3 / 4 \neq 0$. This completes the construction for the Neumann problem.

Remark 3.1. (1) The above construction is related to the construction of non-selfdual Yang-Mills connections over $S^{3} \times S^{1}$ or $S^{4}$. See [5], [9] and [16].

Our above examples give the first examples of non-minimal Yang-Mills connections for the Dirichlet problem and for the Neumann problem with prescribed class $\eta(P)$ (in our case $\eta(P)=0$ ), since flat connection is the only minimal solution for the Dirichlet problem with flat boundary condition (see [4]) and for the Neumann problem with prescribed class $\eta(P)=0$.

(2) It is obvious from (3.1) that the connections constructed above are irreducible connections.

From Theorem 2.1, Theorem 2.2 and these examples, we can conclude that the existence of non-flat Yang-Mills connections for the Dirichlet and Neumann problems depends on the geometry of $M$.

It would be of interest to investigate the effect of the geometry (or topology) of $M$ for the existence of the solutions to boundary value problems of Yang-Mills connections. Such relations are established for the existence problem of (anti-)selfdual connections over closed 4-manifolds, see [12] and [13]. For similar problems related to Yamabe equation and other semi-linear elliptic equations, see [2] and [3].

\section{ACKNOWLEDGEMENTS}

The author wishes to express his gratitude to the referee for many helpful comments and suggestions on an earlier version of this paper. Without his/her help, we would not have this paper in the present form. The author also wishes to thank Professor Antonella Marini for her interest in this work.

\section{REFERENCES}

1. N. Aronszajn, A unique continuation theorem for solutions of elliptic partial differential equations or inequalities of the second order, J. Math. Pures Appl. 36 (1957), 235-249. MR 19:1056c

2. A. Bahri and J. M. Coron, On a nonlinear elliptic equation involving the Sobolev exponent: the effect of the topology of the domain, Comm. Pure Appl. Math. 41 (1988), 253-294. MR 89c:35053

3. H. Brezis, Elliptic equations with limiting Sobolev exponents - The impact of topology, Comm. Pure Appl. Math. 39 (1986), S17-S39. MR 87k:58272

4. T. Isobe and A. Marini, On topologically distinct solutions to the Dirichlet problem for YangMills connections (to appear).

5. H. T. Laquer, Stability properties of the Yang-Mills functional near the canonical connection, Michigan Math. J. 31 (1984), 139-159. MR 88a:58047

6. L. Lemaire, Applications harmoniques de surfaces Riemanniennes, J. Diff. Geom. 13 (1978), 51-78. MR 80h:58024

7. A. Marini, Dirichlet and Neumann boundary value problems for Yang-Mills connections, Comm. Pure Appl. Math. 45 (1992), 1015-1050. MR 93k:58059

8. R. S. Palais, The principle of symmetric criticality, Comm. Math. Phys. 69 (1979), 19-30. MR 81c:58026

9. T. Parker, Non-minimal Yang-Mills fields and dynamics, Invent. Math. 107 (1992), 397-420. MR 93b:58037

10. S. Pohozaev, Eigenfunctions of the equation $\Delta u+\lambda f(u)=0$, Soviet Math. Dokl. 6 (1965), 1408-1411.

11. P. Price, A monotonicity formula for Yang-Mills fields, Manuscripta. Math. 43 (1983), 131166. MR 84m:58033 
12. C. H. Taubes, Self-dual connections on non self-dual 4-manifolds, J. Diff. Geom. 17 (1982), 139-170. MR 83i:53055

13. _ Self-dual connections on 4-manifolds with indefinite intersection matrix, J. Diff. Geom. 19 (1984), 517-560. MR 86b:53025

14. U_ Unique continuation theorems in gauge theories, Comm. Anal. Geom. 2 (1994), 3552. MR 95j:58032

15. K. Uhlenbeck, Connections with $L^{p}$ bounds on curvature, Comm. Math. Phys. 83 (1982), 31-42. MR 83e:53035

16. H. Urakawa, Equivariant theory of Yang-Mills connections over Riemannian manifolds of cohomogeneity one, Indiana Univ. Math. J. 37 (1988), 753-788. MR 90h:53033

17. H. Wente, The differential equation $\Delta x=2 H x_{u} \wedge x_{v}$ with vanishing boundary values, Proc. Amer. Math. Soc. 50 (1975), 131-137. MR 51:10871

Department of Mathematics, Faculty of Science, Tokyo Institute of Technology, OH-OKAYAMA, Meguro-Ku, TOKYO 152, JAPAN

E-mail address: isobe@math.titech.ac.jp 